\title{
Del conocimiento teórico contemplativo al saber dominador y destructor
}

\section{From the theoretical contemplative knowledge to the domineering and destructive knowledge}

Raúl Fornet Betancourt ISIS-Institut e.V., Alemania

Resumen: En este artículo planteamos el tema de una ruptura epistemológica al interior de la historia intelectual europea. El paso de un saber teórico contemplativo, a un saber técnico dominador. Cambio que tuvo implicaciones no sólo en el terreno de la ciencia, sino también en la economía y en la política. La reflexión acerca del cambio de orientación la hacemos a la vista de un diálogo intercultural, el cual propone la recuperación de conocimientos vivenciales como sabiduría alternativa presente en muchas culturas.

Palabras clave: saber teórico, saber técnico, ruptura epistemológica, diálogo intercultural.

Abstract: In this article, we raise the issue of an epistemological rupture within European intellectual history. The passage from a theoretical contemplative knowledge, to a dominating technical knowledge. Change that had implications not only in the field of science, but also in economics and politics. The reflection on 
the change of orientation is made in the light of an intercultural dialogue, that proposes the recovery of experiential knowledge as alternative wisdom present in many cultures.

Keywords: Theoretical knowledge, Technical knowledge, Epistemological rupture, Intercultural dialogue.

\section{Observación preliminar}

G ste artículo propone discutir el tema "Saberes múltiples en las Ciencias Sociales y Políticas" ${ }^{1}$ en el marco del debate actual entre las posiciones del monismo y del pluralismo epistemológicos. Como aportación a la discusión de este tema, presentamos algunas reflexiones sobre lo que a nuestro modo de ver constituye la ruptura epistemológica más radical y fundamental que se ha producido en el interior mismo de la historia intelectual europea y que, por ello, significó un cambio tanto en la propia percepción de Europa como en su relación con otras culturas. Nos referimos, como se desprende del título, al corte que marca el paso de un saber 'teórico', contemplativo, a un saber 'técnico', dominador y reductor, que será a su vez la base del 'camino especial' de Occidente, como veremos luego.

Sin embargo, en el planteamiento de esta cisura trataremos de hacer ver también que ello no es simplemente un hecho relevante para la historia de la ciencia sino también, y acaso fundamentalmente, un acontecimiento político de primer orden en cuanto que el 'monismo epistemológico', justo desde esa cisura se convierte en virulentamente militante, se entiende como un pilar imprescindible en el proyecto de la nueva formación económica entonces

${ }^{1}$ El presente trabajo es la versión escrita y aumentada de una ponencia leída en el coloquio que, bajo el tema homónimo, organizó la Universidad Nacional de Colombia del 18 al 21 de octubre del 2016 en Bogotá. 
naciente y de la civilización que en ella se asentará. De tal manera que, parafraseando la famosa sentencia de Antonio de Nebrija en el prólogo de su gramática castellana "Siempre la lengua fue compañera del imperio" (1980: 97), podríamos asentar que "siempre el monismo epistemológico fue compañero del imperio". Pero no adelantemos nuestras reflexiones, y volvamos a su intención o propósito para decir más bien algunas palabras sobre el punto de partida o el trasfondo filosófico que suponen, que es el verdadero fin de esta observación preliminar.

En este sentido, aclaramos, pues, que la lectura crítica de la cisura, mencionada como intención de estas reflexiones, descansa sobre varios supuestos o convicciones que conforman su verdadero punto de partida y que, como tal, son la fuente de inspiración para la posición que sometemos a la discusión. Así, suponemos, en primer lugar, que el orden del saber que llamamos orden epistemológico no agota el ámbito cognitivo humano. La epistemología no es la medida ni el ideal único del conocimiento humano. Dicho en otros términos: suponemos que no todo saber o, mejor dicho, conocimiento es 'ciencia', sobre todo cuando se toma este término en su acepción hegemónica actual que lo limita por lo general a la connotación de nombre inglés de science (Cf. Snow, 1959; Habermas, 1976: 104ss.).

De lo anterior suponemos, en segundo lugar, que plantear el tema en términos de un debate epistemológico entre el monismo y el pluralismo epistemológicos es, ciertamente, necesario; pero pienso que ello no es suficiente. ¿Por qué? A mi parecer, este planteamiento corre el peligro de reducir el debate a una discusión entre saberes que tienen la pretensión de ser 'científicos' o que son reconocidos como tales por su pertenencia a ciertos lugares y/o métodos de producción de saber vinculados con disciplinas académicas creadas precisamente por el orden epistemológico hegemónico. Queremos decir: desde un horizonte abierto por experien- 
cias interculturales el desafío que plantea este debate de 'saberes múltiples', se entiende como un debate mucho más radical; esto porque, si tomamos en serio la 'multiplicidad' a la que se alude, de lo que se debe tratar es del posible diálogo de esos saberes 'epistemológicos' con aquellos otros conocimientos que no se denominan 'epistemológicos' ni les interesa ser reconocidos como tales. Es decir, que en este debate se tendría que ir más allá de las demandas de un 'equilibrio epistemológico' o de la 'justicia epistemológica', como se ha sostenido en otros estudios (Fornet-Betancourt, 1998: 8-19); (2006: 91-104; 2009: 9-26). Suponemos, por tanto, y en resumen, que hay que ser más radicales en el planteamiento, es decir, hay que poner en cuestión la pretensión de universalidad incontestable del concepto mismo de epistemología, para ver no sólo la carga de eurocentrismo que refleja sino para destapar también la violencia que implica y la humillación que significa para otros conocimientos. $\mathrm{Al}$ hacer este cuestionamiento, nos ponemos alerta también contra la posible trampa que puede implicar el intento de renovarlo tratando de pensarlo más pluralmente (pues, el hacerlo puede ser un esfuerzo engañoso, si su pluralización se sigue pensando desde el horizonte de la misma cultura científica que lo ha expandido).

Suponemos, en tercer lugar, que la división epistemológica y metodológica que separa hoy a la ciencia según la dicotomía de ciencias de la naturaleza y ciencias de la ingeniería, por una parte, y de ciencias humanas o del espíritu, por otra parte, es el reflejo de una falsa precomprensión de la naturaleza como un ámbito de realidad al que el hombre no pertenece o, dicho desde la otra perspectiva, de una falsa comprensión del hombre como 'sujeto' desvinculado del orden natural. Suponemos, pues, que en esa división de la ciencia (que sostiene y legitima, por cierto, la misma organización y profesionalización de los saberes en las facultades universitarias de hoy) se refleja una separación entre hombre y naturaleza y/o 
entre naturaleza y sociedad que bloquea una comprensión integral de la realidad (Cf. Klaus Michael Meyer-Abich, 1997: 72ss.).

Suponemos, en cuarto lugar, que con esta clasificación y división de la ciencia se consolida la fractura cultural que significó, en la influencia social y en los mundos de la vida, la conocida doctrina centroeuropea moderna de las dos sustancias, la res extensa y la res cogitans. Desde este momento, habrá que diferenciar, justamente con una diferencia sustancial, entre cosmología y antropología. Y esta escisión significa que en la cultura que la encarna no hay 'comunicación' vital entre la naturaleza y/o el cosmos y el hombre. Con la naturaleza no se habla, pues ésta sólo se explota, se trabaja e investiga.

Suponemos, en quinto lugar, y en vinculación con lo anterior, que en el fondo de esta concepción hegemónica de la ciencia se hallan el dualismo sujeto-objeto así como el agresivo antropocentrismo, el cual se deriva del mismo como instrumento del homo faber para extender su radio de fabricación.

Y suponemos, en sexto y último lugar, que el dispositivo científico y tecnológico hegemónico, en nuestro presente histórico, no solamente es represivo en el nivel cognitivo -en el sentido, por ejemplo, de que regatea la legitimidad de los conocimientos alternativos con el sólo argumento de que no son 'científicos'- sino también en el ámbito social y político, en tanto que funciona como un aparato institucional que estabiliza el status quo de las sociedades contemporáneas y oprime formas de vida alternativas, como denunciara ya Herbert Marcuse (1968), entre otros.

Bien, sobre el telón de fondo de estos supuestos, y de acuerdo a la intención central de la contribución que queremos hacer al debate en este tema, estructuramos el presente texto según los pasos siguientes:

- El 'camino especial' de la racionalidad europea moderna como conflicto, ruptura y construcción de hegemonía. 
- Consecuencias para el debate intercultural

2. El 'camino especial' de la racionalidad europea moderna como conflicto, ruptura y construcción de hegemonía

Por lo general, los estudiosos de la historia de la ciencia y de la cultura europeas suelen asociar el comienzo de lo que, siguiendo los análisis de Max Weber (1988; 1993), se ha dado en llamar "el camino especial y propio", el cual sigue el Occidente moderno y por el cual éste establece una diferencia de fondo respecto del desarrollo social y cultural de otros continentes, con la emergencia de la ciencia moderna, entendida ésta en el sentido preciso de la formación de un modo de pensar que pone en marcha una racionalidad centrada en el análisis diferencial, en la precisión de sus métodos de medir y calcular, en la cuantificación de lo real así como en la matematización y formalización de los resultados de su procedimiento analítico.

Conocidos son los nombres a los que normalmente se recurre como testigos natos del nuevo comienzo de una ciencia experimental y técnica; por ejemplo: Nicolás Copérnico (1473-1543), Galileo Galilei (1571-1642), Francis Bacon (1561-1626), Johannes Kepler (1571-1630) o Isaac Newton (1642-1726). Igualmente conocido es el dato, importante en este contexto, que significó el pronto respaldo social y político que recibió esta nueva ciencia moderna por parte del poder político de su tiempo, como se comprueba por ejemplo con la fundación en 1660 de la Royal Society of Science en Londres, la cual toma como lema la expresiva fórmula de Nulluis in verba y que de acuerdo con ella declara su convicción rectora de no aceptar como saber ninguna idea que no esté basada y respaldada por hechos determinados como tales por la vía del experimento (Seele, 2008: 54ss.). 
En este contexto debemos recordar además que, siguiendo también la interpretación de Max Weber, el rápido y potente desarrollo de la ciencia europea moderna se suele conectar con la emergencia y la expansión de la formación económica capitalista. ${ }^{2}$

Bástenos aquí esta breve indicación histórica sobre el comienzo del "camino especial y propio" de Occidente para ilustrar el contexto del dato sobre el que queremos reflexionar en este apartado, a saber, que ese llamado "camino especial y propio" de la racionalidad occidental moderna nos confronta con un acontecimiento cuya importancia histórica brota de la conjugación en ella de una novedad doble: la novedad de la ciencia experimental y técnica, por una parte, y la novedad de la formación social y económica que marca la emergencia del capitalismo, por otra parte.

Frente a este dato, y fijándonos sólo en la novedad epistémica, nos preguntamos: ¿Qué tipo de novedad es ésta? Nuestra respuesta sería la siguiente: no se trata ni de una novedad que inventa desde la nada ni de una novedad que innova por el progreso desde el pasado heredado, sino que estamos más bien ante una novedad que debemos interpretar como ruptura que se impone en una confrontación conflictiva con y en la tradición cultural donde acontece. En concreto, a esta ruptura nos referimos cuando en el título de este texto hablamos del cambio de rumbo que implica el giro del conocimiento contemplativo al saber dominador. Con ello, pues, no hablo de un paso que se da como resultado de una transición por evolución sino justo como una ruptura conflictiva en el inte-

${ }^{2}$ Ver, además de los trabajos conocidos de Max Weber, la obra de Edgar Zilsel (1976). Consultar además los detallados análisis sobre la compleja interacción entre cambios de mentalidad o de concepciones científicas y cambios sociales que presentados recientemente en la obra de Arno Bammé (2011) en cuyo título por cierto nos inspiramos para el título de este trabajo. 
rior de la historia europea misma. Unas breves palabras sobre ello para comprender mejor la radicalidad del corte cultural provocada con este cambio de rumbo que nos lleva de la contemplación a la dominación: la racionalidad que se impone con la ciencia moderna europea, que Theodor W. Adorno y Max Horkheimer -corrigiendo a Max Weber- calificaron de racionalidad instrumental (Cf. 1971; ver también Peña Cabrera, 2005: 37-46), no está en línea de continuidad con los dos pilares fundamentales que hasta ese momento la tradición científica de la cultura europea sostenía; es decir, ni con el pensamiento filosófico griego ni con la cultura cristiana de la Edad Media.

Es cierto que el cristianismo, con su fe en un Dios creador ex nihilo, trascendente y libre con respecto a su creación, contribuye al proceso que luego en la modernidad se llamará la 'secularización' de la realidad, y que con ello fomenta también al 'desencantamiento’ de las fuentes de la inteligencia de la razón humana. Pero es innegable que el cristianismo, como cultura de sentido último para lo real, sigue compartiendo con la herencia de la filosofía griega clásica la concepción de la ciencia como una dimensión especifica de la cultura; esto es, como una actividad de inteligencia integrada en la cultura como práctica de vida de la conciencia del ser humano como viviente que pertenece a un orden de plenitud superior, fijo, estable, eterno: el cosmos, en el pensamiento griego, o Dios, en el horizonte de la cultura cristiana. Esta visión de la ciencia (como actividad racional con conciencia de su pertenencia orgánica al orden de la vida cultural en el sentido indicado) tiene como consecuencia que ésta no pueda ni deba ser una construcción aislada con pretensión de autonomía absoluta. Justo en tanto que la dimensión orgánica de la cultura la ciencia está ordenada por los fines últimos de sentido de ésta y su articulación como saber debe, por tanto, estar al servicio de esos fines últimos de sentido. Y no carece de importancia señalar, aunque tenga que ser sólo de 
pasada, que la ciencia europea, al igual que en otras culturas (como por ejemplo las culturas de la China, la India, del mundo árabe, del mundo andino o maya), es entendida aquí como una flor más en basto jardín de la cultura en general, y que, por consiguiente, se la cultiva como tal, pero sin hacer de ella el núcleo para construir una visión exclusivamente 'científica' del mundo y a merced de los intereses del hombre (Friedrich von Weizsäcker, 1977: 92). Pero volvamos al punto.

Esa comprensión de la ciencia (como actividad de una cultura ordenada a la manifestación de fines últimos como caminos hacia el sentido integral de la realidad en su totalidad) se concretiza, además, en la consecuencia de que ni en el pensamiento griego ni en el cristiano se pueda vincular la ciencia a una idea como la del progreso, en el sentido moderno de este término, o a un programa de investigación indefinido que nunca conoce el momento del 'reposo' contemplativo del conocimiento, el cual ha alcanzado lo que necesita y le basta no para satisfacer ya su búsqueda e inquietud de plenitud, pero sí para sentir la orientación fundamental que deben seguir. ${ }^{3}$

El corte radical que introduce la 'racionalidad instrumental', la cual se impone con la ciencia moderna en relación con la ciencia clásica europea (por llamarla así a efectos de brevedad), significa, por tanto, mucho más que una ruptura metodológica o la simple sustitución de un método intuitivo y deductivo por un método deductivo, analítico y experimental. Este giro metodológico se hace necesario más bien como la secuencia lógica de otro cambio de mayor calado. Nos referimos a la transmutación del ideal del conocimiento humano que está en el trasfondo de este corte; a nuestro modo de ver, éste es el cambio que realmente provoca una ruptura

${ }^{3}$ Para una detallada presentación de las diferencias entre el concepto de ciencia en Platón y Aristóteles, como casos ejemplares para la visión clásica griega, y la ciencia moderna ver Böhme (1993: 81-120). 
en la cadena de la tradición científica europea. Para ilustrar este punto, para nosotros de importancia central, retomaremos ahora la idea del progreso que hemos esbozado antes.

Tanto en la filosofía griega antigua como en el pensamiento medieval cristiano, se conoce el término 'progreso', aplicado incluso a la actividad cognitiva del ser humano. Sin embargo, con ese término se designa sobre todo un crecer orgánico, un proceso en el ser para alcanzar la madurez o, si se prefiere, un camino de perfección, donde es decisivo que ese 'progreso' se cumpla como 'proceso' al interior de los límites del orden cósmico o de la creación (Cf. Ritter, 1972: columna 1032ss; ver también Rapp, 1992). Tiene, pues, el límite del sentido del orden que lo funda como tal. Y de ahí, como decíamos arriba, que pueda conocer el 'reposo'.

Muy distinta es, sin embargo, la concepción del progreso científico que Francis Bacon desarrolla tanto en su obra Proficience and Advencement of Lerning, de 1605, como en su más comentada obra Novum organum scientiarum, sive Indicia vera de interpretatione natura et regno hominis, de 1620. Puesto que, apoyándose justo en el impacto de los nuevos "inventos mecánicos" -como significativamente los llama ${ }^{4}$, Francis Bacon interpreta su época como un tiempo en el que, al derrumbarse con esos inventos las fronteras que hasta entonces se consideraban infranqueables para el hacer del hombre, el saber humano se ve relanzado hacia un horizonte de innovación técnica cuya novedad no solamente resulta inexplicable desde los conocimientos de la tradición sino que invalida incluso la autoridad de los mismos. El progreso, como impulso hacia lo nuevo desconocido, es una carrera en la que el saber tiene que procurar siempre tomar la delantera, por lo tanto, no puede detenerse ni respetar fronteras. Al progreso le es inherente traspasar todo límite. Lo que equivale a decir que la experiencia de

${ }^{4}$ Francis Bacon se refiere en concreto a los descubrimientos geográficos, a la pólvora, al compás y a la imprenta (1962: 129). 
lo real, como 'orden' inteligible, se sustituye por el programa de fabricación ilimitada de cosas. En este marco, el saber científico conlleva en sí una necesidad de progreso, de dejar atrás todo lo antiguo. Pero en este punto conviene prestar atención al subtítulo del Novum organum, pues contiene una importante indicación, una que ayuda a comprender que la necesidad de progreso que conlleva el saber en esos nuevos tiempos de Bacon se asienta en el fondo en el proyecto de instaurar ese "reino del hombre" del que nos habla el subtítulo; un reino que se entiende en términos de un proyecto antropocéntrico de dominio ilimitado de la naturaleza como pilar de una nueva civilización.

Nos permitimos subrayar la conexión de la idea del progreso científico moderno con el proyecto de una nueva civilización, porque pensamos que es precisamente esta relación interna entre ambos lo que ha llevado a que la ciencia europea moderna haya podido construir y consolidar su hegemonía de una forma tan contundente como lo ha hecho y lo hace hasta hoy. Es decir: sin la voluntad política de querer una civilización del progreso técnico e industrial no se explica por qué justamente esta ciencia experimental y tecnológica es considerada como la ciencia que es necesaria no sólo para Europa sino también para todos los pueblos que quieran salir del 'subdesarrollo'. Es este marco civilizatorio lo que impulsa y mantiene su hegemonía, también lo que hace que la idea del 'progreso científico' sea hoy un potente factor de dominación y marginación de otros conocimientos. ${ }^{5}$

${ }^{5}$ Científicos de distinta orientación, como Mario Bunge o Carl Friedrich von Weizsäcker, por ejemplo, coincide en destacar esa conexión entre la ciencia moderna europea y la civilización del progreso característica de Occidente (Cf. Mario Bunge, 1966; Friedrich von Weizsäcker, 1977). Del mismo modo, filósofos como Heidegger han insistido por su parte en que es el modelo de una sociedad industrial lo que ha impulsado esa "necesidad" de la "ciencia objetiva" (Cf. Heidegger, 2009). 
Hasta ahora, he acentuado el conflicto y corte con la tradición europea del pensamiento griego antiguo y el pensamiento cristiano medieval. Pero no entenderíamos bien la complejidad de la tradición europea si no tuviésemos en cuenta también que ese conflicto no es sólo una 'querella entre antiguos y modernos', como a veces se presenta. El conflicto es igualmente, y no en menor medida, un conflicto entre 'modernos'. Porque la historia intelectual europea muestra de manera equivalente que la ciencia experimental del llamado espíritu matemático y analítico es cuestionada por otros modernos; es decir, por pensadores y científicos que buscan también vías modernas, pero que consideran que éstas no pueden ser las propuestas por una visión mecánica y objetivista de la realidad en cuyo horizonte no cabe más que construir relaciones externas entre objetos. Recordemos entre estos modernos alternativos que encarnan las otras caras posibles de la modernidad europea, por ejemplo, a Gottfried Wilhelm Leibniz (1646-1716) con su proyecto de una 'ciencia universal' orgánica que armonice en una nueva síntesis entre razón y misterio la separación 'moderna' de las ciencias de la res extensa y de la res cogitans (Andreu, 2001); proyecto alternativo que resonará todavía en pleno siglo XX con la concepción de la cosmología orgánica que se desarrolla en la obra del matemático y filósofo Alfred North Whitehead (1861-1947) ${ }^{6}$ o en la obra del jesuita y paleontólogo Teilhard de Chardin (1881-1955), con su reclamo de una radical inversión del método científico y con ello de una conversión de la 'Ciencia' hegemónica.'

${ }^{6}$ De la obra de Whitehead ver: El concepto de naturaleza (1968), Science and the Modern World (1925) y Process and Reality. An Essay in Cosmology (1929). Y para una presentación de su proyecto cosmológico en comparación con la ciencia moderna ver: Alois Rust, Die organismische Kosmologie von Alfred $N$. Whitehead (1987).

7 De Theilhard de Chardin ver: La energía humana (1963), La aparición del hombre (1964), El medio divino (1966) y El fenómeno humano (1967). 
Igualmente, recordemos, como ejemplo de la reacción del llamado movimiento 'romántico', a Johann Gottfried Herder (17541803) con su interpretación pluralista e integral de la naturaleza frente a las tendencias antropocéntricas y androcéntricas que se imponían en la ciencia moderna de este tiempo (Herder, 1982); también, por poner otro ejemplo representativo, a Johann Wolfgang von Goethe (1749-1832) con su decidido rechazo al subjetivismo moderno y su desarrollo de una compleja teoría alternativa de los colores ('Farbenlehre'), la cual tenía como intención expresa la corrección de la explicación meramente física elaborada por Newton (Goethe, 1810).$^{8}$

Y podríamos todavía citar otros ejemplos, como la filosofía especulativa de la naturaleza de Friedrich Wilhelm Schelling (17751854) (Cf. Schelling, 1972: 1-268/269-326/635-659), pero creemos que los ejemplos nombrados son suficientes para presentar las caras alternativas que pudo tener la modernidad europea en el campo específico del saber científico. Con ello, esta historia marginada nos hace ver al mismo tiempo que el curso central de la ciencia moderna europea debe ser leído como una construcción de hegemonía ${ }^{9}$ en el marco de un proyecto determinado de civilización y como parte integrante imprescindible de ese modelo como modo de vivir y habitar la tierra.

Esto nos permite aclarar, por otra parte, que lo que desde una perspectiva intercultural se critica nada tiene que ver con residuos de oscurantismo en culturas que todavía no han entrado de lleno en 'el progreso de las luces' o se resisten tercamente a seguir

\footnotetext{
${ }^{8}$ Para una visión documentada de las posibilidades alternativas que tuvo la modernidad europea se puede consultar: Gernot Böhme (1993); Klaus Michael Meyer-Abich (1997a; 1997b).

${ }^{9}$ En este punto resultan sumamente instructivos, desde una perspectiva europea autocrítica, los análisis de los lazos entre la construcción de saber hegemónico y el poder en la obra de Michel Foucault.
} 
dicho camino; culturas que, no es superfluo recordarlo, la ciencia moderna califica con arrogancia de 'subdesarrolladas'. No se trata, por tanto, de ningún afecto anticientífico ni mucho menos antiracional, como tampoco de una expresión de resentimiento ante el 'éxito' espectacular de 'los del Norte'; pues el fundamento y la motivación de la crítica intercultural se alimenta de la idea de que la hegemonía epistemológica es una construcción y que, como en toda construcción de hegemonía, hay reducción, estrechez de miras, dominio y exclusión. Esto no sólo de cara a los saberes de aquellos 'otros' pueblos que no pertenecen al mismo ámbito cultural, sino también de cara a la disidencia epistemológica interna. Dicho con palabras más concretas: la perspectiva intercultural critica a la ciencia moderna europea. Sin embargo, debe quedar claro que no la critica porque sea ciencia ni porque sea moderna ni porque sea europea; al contrario, el propósito fundamental de la crítica intercultural está en mostrar que la ciencia moderna europea es, como ciencia, pobre, como moderna, deficiente y, como europea, reduccionista. Los ejemplos aducidos de las alternativas que su historia dominante ha condenado a la periferia, lo ilustran.

\section{Consecuencias para el debate intercultural}

En este apartado tomaremos como punto de partida de nuestras consideraciones críticas una afirmación de Karl Jaspers (18831969), la cual expresa con claridad casi brutal el verdadero 'secreto' de la hegemonía mundial de la ciencia europea moderna y que, a nuestra manera de ver, sigue siendo tan válida como en el momento en que la escribió, si no es que ha ganado en actualidad. Ahí, Jaspers constata lo siguiente:

El origen de la ciencia y la técnica está en los pueblos germanorrománicos. Estos pueblos han producido con ellas una ruptura histórica. Ellos son los que han iniciado la verdadera historia 
universal, planetaria, de la humanidad. Solo los pueblos que se apropian la ciencia y la técnica occidentales y aceptan los peligros para el ser del hombre, enlazados con este saber y poder, pueden colaborar todavía activamente en la historia (1965: 91-92). ${ }^{10}$

Estas palabras expresan con toda la claridad deseable que el 'secreto' de la hegemonía de la ciencia y la técnica occidentales, sobre todo hoy cuando se expanden en la figura unificada de la tecnología, radica en que se han impuesto como la condición básica para que el hombre del mundo moderno, hoy diríamos 'global', pueda acceder a la realidad e intervenir en su curso de forma históricamente relevante. Pero de esta constatación se desprende, como ya hemos dado a entender en el apartado anterior, que el impacto planetario del que nos habla Jaspers nos es comprensible si no se supone el proyecto político de construir una civilización del progreso industrial que mide, en lo decisivo, el avance de su desarrollo justamente por el grado alcanzado en el dominio tecnológico de la naturaleza y por el consiguiente aumento en la racionalización de la vida. De este modo, podemos afirmar que ese modelo de civilización y ese modelo de ciencia tecnológica se necesitan mutuamente.

De este hecho, y de cara a la crítica intercultural que anima las consideraciones de este apartado, destacamos un primer aspecto, a saber: que si la 'necesidad planetaria' de la ciencia y la técnica occidentales representa un acontecimiento histórico, estando además ligada a la expansión colonialista de un determinado proyecto de civilización, se trata entonces de una necesidad que es contin-

${ }^{10}$ Aquí, resulta interesante que Jaspers, al ubicar el comienzo de la ciencia y la técnica occidentales en los pueblos 'germanorrománicos', está sosteniendo al mismo tiempo que ese origen poco o nada tiene que ver con Grecia. Lo dice expresamente unas páginas más adelante: "El elemento verdaderamente nuevo, fundamentalmente distinto por completo, incomparable con lo asiático, en absoluto independiente, incluso entraño a los griegos, es únicamente la ciencia y la técnica europea moderna” (1965: 113). 
gente, tanto en su origen como en su futuro. Y si es contingente significa que puede ser invertida en el curso real por el que hoy se impone como tal o que, por lo menos, se la puede relativizar o regionalizar en su alcance 'planetario'. Sin embargo, y esto también debemos reconocerlo por honestidad con nosotros mismos, para que esta contingencia sea aceptada (y sobre todo 'querida' en sus consecuencias teóricas y prácticas), hace falta sentir y pensar la pluralidad del mundo como puja cultural por ese otro proyecto alternativo de universalidad sin hegemonía que los zapatistas, por ejemplo, han concretizado en la demanda de un mundo 'colorido' en el que quepan muchos mundos. Es decir, hace falta sentir y vivir la pluralidad del mundo como exigencia de libertad frente al curso totalitario que impone la civilización tecnológica y las pretendidas 'necesidades humanas' que de ahí se desprende.

La crítica intercultural comienza en este nivel no por la demanda de más espacios culturales propios dentro del marco de la civilización hegemónica, sino que va a la raíz del problema y reivindica el derecho de ser libres frente a la civilización actual. Esta libertad de civilización representa la condición primera para que en un diálogo intercultural, específicamente entre las diversas culturas de conocimiento de los pueblos del mundo, se puedan sacar las consecuencias alternativas de lo que hemos llamado la contingencia de la ciencia moderna europea y la civilización que la tiene como su núcleo fuerte.

Pero, ¿queremos realmente esta libertad de civilización para invertir la espiral de un progreso depredador y buscar caminos de plenitud en la diversidad cultural de la humanidad?

Muchas iniciativas de regeneración del mundo y de la vida animan a responder esta pregunta de manera positiva. Con todo, si miramos el 'espectáculo' de nuestro presente histórico, ${ }^{11}$ no parece

${ }^{11}$ Sobre esto se pueden ver las reflexiones del Dr. Raúl Fornet-Betancourt en "Pensar la época" (2016: 129-138). 
que esa posible respuesta positiva sea hoy una evidencia irrefutable. Baste recordar, en un ámbito general, nuestros hábitos de consumo y, en un campo más específico, el sometimiento de nuestra actividad académica a la cultura hegemónica, con la aceptación en nuestras propias prácticas, por ejemplo, de los criterios de la llamada 'excelencia académica' que define el sistema. Sin embargo, debido a que no es evidente que podamos responder positivamente ante la pregunta planteada, la crítica intercultural destaca un segundo aspecto que tiene que ver con la transformación interior que ha provocado la hegemonía de la civilización occidental capitalista en el hombre y que ayudaría a comprender la ambivalencia en que pensamos y vivimos. Lo explicamos brevemente.

Creemos que sería quedarse a mitad de camino en el planteamiento de una crítica radical a la situación en la que está hoy civilizatoriamente la humanidad, asumir que, con sus tecnologías y ofertas de consumo ligadas a un mercado que incrementa la aceleración de la fecha de caducidad de los productos y con ello la creación de nuevas 'necesidades' (por nombrar aquí sólo estos casos), la civilización hegemónica cambia únicamente la faz de la tierra, sin tocar las formas de comprender la vida misma y sus fuentes de sentido en el ser mismo del hombre.

Por lo anterior, proponemos hacernos cargo también del hecho de que, además de ese cambio de la faz de la tierra, estamos ante una transformación del ser humano (Ver Fornet-Betancourt, 2013: 11-24). Esta transformación, que hemos llamado interior porque tiene lugar en el mismo ser humano, se puede explicar como consecuencia de muchos y diversos factores, bien estructurales -como serían las complejas estrategias de integración en el sistema hegemónico- o personales -como serían opciones de vida individual inspiradas en la 'mala fe', para decirlo con este término del Sartre fenomenólogo (1973: 85)-. Pero, en el contexto de nuestra argumentación, lo decisivo es fijarse en que esta transfor- 
mación interior se manifiesta esencialmente como un cambio en la estructura de atención del conocimiento humano; el cual implica a su vez un cambio en el carácter del conocimiento, en su finalidad $y$, por consiguiente, también en su relación con la vida. ¿’Por qué? Porque la participación del hombre en esa maquinaria tecnológica que proyecta el mundo 'global', como un campo de progreso ilimitado que reclama 'estar a la expectativa' de los avances programados, lleva a que la atención del conocimiento se desvíe justamente hacia la espera de las novedades y hacia un saber que le informe lo más pronto posible sobre las nuevas ofertas. La atención del conocimiento se vuelca así en la expectativa de lo nuevo, y como la secuencia de lo nuevo se anuncia cada vez con mayor celeridad, esto significa la dispersión y la disolución de la atención en un permanente y desasosegado estar a la caza de informaciones, con la consecuencia extrema de que en esta situación el hombre 'pierde el conocimiento'. Pero ¿qué queremos decir con esto?

Esta 'pérdida de conocimiento', como momento culminante de la transformación interior que explicamos, no la entendemos como un resultado del deslumbramiento o aturdimiento que puede provocar en el hombre la rápida secuencia de las expectativas de lo nuevo o el torbellino de las 'informaciones'. La comprendemos más bien, y por eso hablamos de transformación interior, como el proceso mediante el cual se sustituye el 'conocer humano contextual', que es conocimiento lento de inteligencia atenta a la vida y sus mundos, por el 'saber funcional de...', que es un saber técnico de información para 'andar por el mundo' global.

Debemos aclarar que suponemos en ella la diferencia que hace la lengua castellana entre conocer y saber respecto a toras lenguas románicas, la cual nos dice que el conocer es una actividad que requiere la experiencia personal, mientras que el saber es un acto impersonal y transmisible indirectamente. Luis Villoro plasma esta diferencia en estos términos: 
En castellano existen dos verbos que no suelen usarse con el mismo significado: 'conocer' y 'saber' [...] Esta distinción semántica puede orientarnos para descubrir otra de importancia epistemológica, que no se ha presentado aún con claridad, debido tal vez a que los principales análisis de los conceptos epistémicos han sido escritos en inglés, lengua en que esta distinción se ha perdido [...] Para conocer algo es preciso haber tenido una experiencia personal y directa, haber estado en contacto, estar 'familiarizado' con ello [...] Saber, en cambio, no implica tener una experiencia directa (1982: 97-99).

Desde la comprensión de la transformación interior y la diferencia entre 'conocer' y 'saber' que ésta supone, la crítica intercultural responde ante la 'pérdida de conocimiento' (que a la larga provoca dicha transformación en la estructura de atención del conocimiento) con la propuesta de un diálogo para 'recobrar el conocimiento'; sin embargo, entiende este diálogo no tanto como un intercambio entre 'saberes' sino más bien como un diálogo entre 'conocimientos' que, en la línea de la 'diferencia epistemológica' apuntada por el filósofo mexicano Luis Villoro, nos comprometa en un intercambio de experiencias personales de 'familiarización' donde se ponga de manifiesto que el conocimiento no es únicamente una actividad cognitiva, 'epistémica', sino un proceso antropológico de participación en lo real que involucra a todo el ser humano. Se trataría de promover, dicho ahora con un término de Orlando Fals Borda, un diálogo entre los 'conocimientos vivenciales' por los que los pueblos humillados por la arrogancia de la academia expresan su 'con-ciencia' de participación en el mundo y muestran, contra todo intento de eurocentrismo por parte de los 'colonos intelectuales' de ayer y de hoy, que el mundo es plural y que ahora se conoce se habita también de forma plural (Fals, 1988: 97-105). 
Además, 'recobrar el conocimiento' por este diálogo de conocimientos vivenciales significaría, en la propuesta intercultural, la condición para lograr la 'con-ciencia' que se necesita para poder discernir nuestra situación éticamente y 'querer' poner en marcha la práctica del derecho a ser libres frente a la civilización hegemónica.

Por último, queremos mencionar un tercer aspecto que plantea la crítica intercultural como otra de las consecuencias que hay que considerar en la confrontación con la civilización hoy hegemónica $y$, específicamente, con la constelación epistemológica de los saberes que ha dibujado. Nos referimos a la cuestión siguiente: en un diálogo intercultural la vuelta recuperadora a los 'conocimientos vivenciales' tiene que superar todo residuo de prejuicios 'ilustrados', para incluir en ese diálogo de conocimientos las tradiciones de espiritualidad que impregnan hasta hoy la sabiduría de vida alternativa de muchas culturas, como, por ejemplo, la sabiduría del Buen Vivir en las culturas andinas de Abya Yala. Este paso nos parece de fundamental importancia, sobre todo de cara a un debate que quiera abrir un nuevo horizonte para el conocimiento humano; puesto que tal vez son las espiritualidades con sus conocimientos sapienciales la reserva que con más radicalidad puede contribuir hoy a reconcentrar la atención de los procesos de conocimiento en lo que es vitalmente necesario -también se puede decir: concentrar la atención en lo que nos falta en verdad, que muy posiblemente sea justo, aunque parezca paradójico, lo que no basta- (Ver Fornet-Betancourt, 2016: 25-38) y romper así la lógica funcional del progreso como hilo conductor del conocimiento o, mejor dicho, saber. ${ }^{12}$

12 Sobre esta cuestión del significado de la espiritualidad en el debate epistemológico hoy ver: Raúl Fornet-Betancourt (2014; 2015). 


\section{Bibliografía}

Andreu Rodrigo, Agustín, 2001, La inteligencia en la torre. Razón y misterio en la Ilustración Leibniziana, Editorial Universidad Politécnica de Valencia, Valencia.

Bacon, Francis, 1962, Novum Organum, I.

Bammé, Arno, 2011, Homo occidentalis. Von der Anaschauung zur Bemächtigung der Welt. Zäsuren abendländischer Epistemologie, Velbrück Verlag, Weilerswist.

Böhme, Gernot, 1993, Alternativen der Wissenschaft, Suhrkamp Verlag, Frankfurt/M.

Bunge, Mario, 1966, La ciencia, su método a su filosofía, Editorial Siglo Veinte, Buenos Aires.

Chardin, Theilhard de, 1963, La energía humana, Taurus Ediciones, Madrid.

, 1964, La aparición del hombre, Taurus Ediciones, Madrid. , 1966, El medio divino, Taurus Ediciones, Madrid. , 1967, El fenómeno humano, Taurus Ediciones, Madrid.

Fals Borda, Orlando, 1988, "Por un conocimiento vivencial", Concordia. Internationale Zeitschrift für Philosophie 12.

Fornet-Betancourt, Raúl, 1998, "Lernen zu philosophieren ausgehend vom Kontext des Dialogs der Kulturen”, en Raúl FornetBetancourt (ed.), Unterwegs zur Interkulturellen Philosophie. Dokumentation des II. Internationalen Kongresses für Interkulturelle Philosophie, Frankfurt, IKO/Verlag, pp. 8-19.

, 2006, "La pluralidad de conocimientos en el diálogo intercultural”, en Raúl Fornet-Betancourt. La interculturalidad a prueba, Verlag Mianz, Aachen, pp. 91-104.

,2009, "El encuentro de las culturas del saber como camino para pensar el saber que deberíamos saber", en Raúl Fornet-Be- 
tancourt, Tareas y propuestas de la Filosofía Intercultural, Verlag Mainz, Aachen, pp. 9-26.

, 2013, "Eine anthropologische Krise? Anmerkungen zu einer kritischen Interpretation der anthropologischen Situation heute", en Raúl Fornet-Betancourt Interkulturalität und Menschlichkeit, Verlag Mainz, Aachen, pp. 11-24.

, (ed.), 2014, Justicia, conocimiento y espiritualidad, Verlag Mainz, Aachen.

, (ed.), 2015, Tradiciones de formación, espiritualidad y universidad, Verlag Mainz, Aachen.

, 2016a, "Notas para una reflexión sobre la pregunta: ¿Qué falta?", en Raúl Fornet-Betancourt, Filosofía y espiritualidad en diálogo, Verlag Mainz. Aachen, pp. 25-38.

, 2016b, "Pensar la época", en Filosofía y espiritualidad en diálogo, Verlag Mainz, Aachen.

Friedrich von Weizsäcker, Carl, 1977, Der Garten des Menschlichen. Beiträge zur geschichtlichen Anthropologie, Verlag Hanser, München-Wien.

Goethe, Johann Wolfgang, 1810, Zur Farbenlehre, dos tomos, Cotta Verlag, Tübingen.

Habermas, Jürgen, 1976, "Technischer Fortschritt und soziale Lebenswelt", en Technik und Wissenschaft als "Ideologie", Suhrkamp. Frankfurt/M.

Heidegger, Martin, 2009, Leitgedanken zur Entstehung der Metaphysik, der neuzeitlichen Wissenschaft und der modernen Technik, en Gesamtausgabe, tomo 76, Verlag Klostermann, Frankfurt/M.

Herder, Johann Gottfried, 1982, Ideen zur Philosophie der Geschichte der Menschheit, en Herders Werke in fünf Bänden, Aufbau-Verlag, Berlin/Weimar.

Horkheimer, Max y Theodor W. Adorno, 1971, Dialektik der Aufklärung, Fischer Verlag, Frankfurt/M. 
Jaspers, Karl, 1965, Origen y meta de la historia, Editorial Revista de Occidente, Madrid.

Marcuse, Herbert, 1968, El hombre unidimensional. Ensayo sobre la ideología de la sociedad industrial avanzada, Editorial Joaquín Mortiz, México.

Meyer-abich, Klaus Michael, 1997, Praktische Naturphilosophie. Erinnerung an einen vergessenen Traum, Verlag C.H. Beck, München.

Nebrija, Antonio de, 1980, Gramática de la lengua castellana, A. Chuitis (ed.), Madrid.

Peña Cabrera, Antonio, 2005, "Racionalidad occidental y racionalidad andina", en Antonio Peńa Cabrera et at., La racionalidad andina, Editorial Mantaro, Lima, pp. 37-46.

Sartre, Jean-Paul, 1973, L'être et le néant. Essai d'ontologie phénoménologique, Éditions Gallimard, Paris.

Schelling, Friedrich Wilhelm, 1972, Erster Entwurf eines System der Naturphilosophie, en Ausgewählte Werke, tres tomos, Wissenschaftliche Buchgesellschaft, Darmstadt.

Seele, Peter (ed.), 2008, Philosophie des Neuen, Wissenschaftliche Buchgesellschaft, Darmstadt.

Snow, Charles Percy, 1959, The Two Cultures, University Press, Cambridge.

Friedrich Rapp, 1992, Fortschritt. Entwicklung und Sinngehalt einer philosophischen Idee, Wissenschaftliche Buchgesellschaft, Darmstadt.

Joachim Ritter 1972, "Fortschritt", en Joachim Ritter et al., Historisches Wörterbuch der Philosophie, t. 2, Schwabe Verlag, Basel.

Rust, Alois, 1987, Die organismische Kosmologie von Alfred $N$. Whitehead, Athenäum Verlag, Frankfurt/M.

Villoro, Luis, 1982, Creer, saber, conocer, Siglo XXI, México. 
Weber, MAX, 1988, Gesammelte Aufsätze zur Religionssoziologie I, J.C.B. Mohr Verlag, Tübingen. , 1993, Die protestantische Ethik und der »Gesit « des Kapitalismus, Syndikat Verlag, Frankfurt/M.

Whitehead, Alfred N., 1925, Science and the Modern World, Macmillan Comp., New York.

1929, Process and Reality. An Essay in Cosmology, Macmillan Comp., New York. , 1968, El concepto de naturaleza, Editorial Gredos, Madrid.

Edgar Zilsel, 1976, Die sozialen Ursprünge der neuzeitlichen Wissenschaft, Suhrkamp Verlag, Frankfurt/M.

Recibido: 17 de agosto de 2017 Aceptado: 2 de noviembre de 2017 